\title{
The distribution of snow black carbon observed in the Arctic and compared to the GISS-PUCCINI model
}

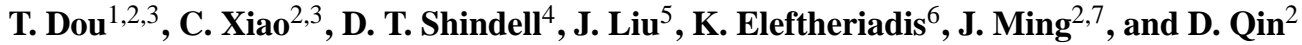 \\ ${ }^{1}$ College of Resources and Environment, University of Chinese Academy of Sciences, Beijing 100049, China \\ ${ }^{2}$ State Key Laboratory of Cryospheric Sciences, Cold and Arid Regions Environmental and Engineering Research Institute, \\ Chinese Academy of Sciences, Lanzhou 730000, China \\ ${ }^{3}$ Institute of Climate System, Chinese Academy of Meteorological Sciences, Beijing 100081, China \\ ${ }^{4}$ NASA Goddard Institute for Space Studies and Columbia Earth Institute, Columbia University, New York, NY 10025, USA \\ ${ }^{5}$ State Key Laboratory of Numerical Modeling for Atmospheric Sciences and Geophysical Fluid Dynamics, \\ Institute of Atmospheric Physics, Chinese Academy of Sciences, Beijing 100029, China \\ ${ }^{6}$ Environmental Radioactivity Laboratory, I.N.Ra.S.T.E.S, National Centre for Scientific Research "Demokritos", \\ 15310 Ag. Paraskevi, Attiki, Greece \\ ${ }^{7}$ National Climate Center, China Meteorological Administration, Beijing 100081, China
}

Correspondence to: C. Xiao (cdxiao@cams.cma.gov.cn)

Received: 21 February 2012 - Published in Atmos. Chem. Phys. Discuss.: 3 May 2012

Revised: 13 August 2012 - Accepted: 29 August 2012 - Published: 7 September 2012

\begin{abstract}
In this study, we evaluate the ability of the latest NASA GISS composition-climate model, GISS-E2PUCCINI, to simulate the spatial distribution of snow BC (sBC) in the Arctic relative to present-day observations. Radiative forcing due to $\mathrm{BC}$ deposition onto Arctic snow and sea ice is also estimated. Two sets of model simulations are analyzed, where meteorology is linearly relaxed towards National Centers for Environmental Prediction (NCEP) and towards NASA Modern Era Reanalysis for Research and Applications (MERRA) reanalyses. Results indicate that the modeled concentrations of $\mathrm{SBC}$ are comparable with presentday observations in and around the Arctic Ocean, except for apparent underestimation at a few sites in the Russian Arctic. That said, the model has some biases in its simulated spatial distribution of $\mathrm{BC}$ deposition to the Arctic. The simulations from the two model runs are roughly equal, indicating that discrepancies between model and observations come from other sources. Underestimation of biomass burning emissions in Northern Eurasia may be the main cause of the low biases in the Russian Arctic. Comparisons of modeled aerosol $\mathrm{BC}(\mathrm{aBC})$ with long-term surface observations at Barrow, Alert, Zeppelin and Nord stations show significant underestimation in winter and spring concentrations in the Arctic (most significant in Alaska), although the simu-
\end{abstract}

lated seasonality of $\mathrm{aBC}$ has been greatly improved relative to earlier model versions. This is consistent with simulated biases in vertical profiles of $\mathrm{aBC}$, with underestimation in the lower and middle troposphere but overestimation in the upper troposphere and lower stratosphere, suggesting that the wet removal processes in the current model may be too weak or that vertical transport is too rapid, although the simulated BC lifetime seems reasonable. The combination of observations and modeling provides a comprehensive distribution of $\mathrm{sBC}$ over the Arctic. On the basis of this distribution, we estimate the decrease in snow and sea ice albedo and the resulting radiative forcing. We suggest that the albedo reduction due to $\mathrm{BC}$ deposition presents significant space-time variations, with highest mean reductions of $1.25 \%$ in the Russian Arctic, which are much larger than those in other Arctic regions $(0.39 \%$ to $0.64 \%)$. The averaged value over the Arctic north of $66^{\circ} \mathrm{N}$ is $0.4-0.6 \%$ during spring, leading to regional surface radiative forcings of $0.7,1.1$ and $1.0 \mathrm{~W} \mathrm{~m}^{-2}$ in spring 2007, 2008 and 2009, respectively. 


\section{Introduction}

Emissions of black carbon (BC) particles result from incomplete combustion during the burning of biomass and fossil fuels, and are considered a significant climate forcing factor (IPCC, 2007; McConnell et al., 2007). In the atmosphere, the absorption of sunlight by BC contributes to global warming and alters cloud-formation processes (Jacobson, 2001). After deposition onto snow and ice, BC has the potential to significantly reduce the surface albedo, hence perturbing the radiative balance and possibly leading to earlier snowmelt (Hegg et al., 2009). The global average radiative forcing from $\mathrm{BC}$ by altering surface albedo was estimated as $+0.1 \mathrm{~W} \mathrm{~m}^{-2}$ (IPCC, 2007), with estimates varying from 0.01 to $0.16 \mathrm{~W} \mathrm{~m}^{-2}$ (Flanner et al., 2007; Hansen et al., 2004; 2007; Koch et al., 2009a).

Arctic climate is especially vulnerable to $\mathrm{BC}$ deposition because of the abundant and wide distribution of relatively clean sea ice, snow and glaciers, which can be impacted effectively by accelerating melting and snow/ice albedo feebacks (McConnell et al., 2007; Koch and Hansen, 2005). Recent research suggests that the seasonally averaged surface forcing by $\mathrm{BC}$ in the Arctic can be up to $+0.5 \mathrm{~W} \mathrm{~m}^{-2}$ in spring (Flanner et al., 2007; Quinn et al., 2008). The comparisons between modeled and observed aerosol $\mathrm{BC}(\mathrm{aBC})$ at Barrow, Alert and Zeppelin stations have shown that most previous models underestimated concentrations of $\mathrm{BC}$ in the Arctic, especially in winter and spring (Shindell et al., 2008; Huang et al., 2010a; Liu et al., 2011). However, the seasonal cycle and magnitude of other trace species, like $\mathrm{CO}$ and ozone, are reproduced much better in most models (Shindell et al., 2008).

There have been many studies on the dominant causes of the present discrepancy between models and observations. Several factors were suggested, such as the uncertainty of the emission inventory used in the model (Wang et al., 2011), the modeling of transport processes (Liu et al., 2011), the lack of significant number of observation sites available to adequately validate models, or the existing challenges in quantifying $\mathrm{BC}$ mass concentrations by current measurement methods introducing an inherent uncertainty on reported BC mass concentrations (Shindell et al., 2008; Doherty et al., 2010; Baumgardner et al., 2012). In recent years, model simulations and measurement methods have been updated and greatly improved, so that it is useful to collate comprehensive pan-Arctic $\mathrm{sBC}$ observations from previous campaigns to re-evaluate the current models. Koch et al. (2009b) compared vertical profiles of aBC from several models to aircraft observations during the International Polar Year (IPY), and suggested that current models underestimated BC concentrations throughout much of the troposphere in different degrees. There still exist large uncertainties in this comparison, because the observations of vertical profiles of $\mathrm{aBC}$ present just a "snapshot" of the $\mathrm{BC}$ distribution in limited sites and they are not representative over the whole Arctic, especially at climate scales. More extensive and long-term observations are still needed to give a comprehensive validation to current models. This study focuses on the latest NASA GISS composition-climate model (hereafter GISS-E2-PUCCINI), aiming to investigate the performance of the simulation of spatial distribution of $\mathrm{BC}$ deposition on Arctic snow and ice. We summarize BC measurements reported to date and add the observations obtained in the first Korean Arctic cruise (2010) to the analysis. We use these to evaluate the modeled distribution of $\mathrm{sBC}$ in the Arctic and then give a comprehensive map of the spring sBC in and around the Arctic Ocean with a combination of observations and validated model results. Finally, we estimate the decrease in snow and sea ice albedo and resulting radiative forcing due to $\mathrm{BC}$ deposition based on the derived $\mathrm{SBC}$ distribution.

\section{Observations}

The earliest observations of sBC started from the mid1980s (Clarke and Noone, 1985), and were carried out at only a few sites, such as Camp Century, Greenland $\left(77.2^{\circ} \mathrm{N}, 61.1^{\circ} \mathrm{W}\right)$, Dye 3 , Greenland $\left(65.2^{\circ} \mathrm{N}, 43.8^{\circ} \mathrm{W}\right)$, Alert, Canada $\left(83.5^{\circ} \mathrm{N}, 62.5^{\circ} \mathrm{W}\right)$, Barrow, Alaska $\left(71.3^{\circ} \mathrm{N}\right.$, $156.6^{\circ} \mathrm{W}$ ) and several Arctic Ocean sites. Later, the spatial distribution of $\mathrm{BC}$ in snow and sea ice was investigated during the SHEBA experiment (Grenfell et al., 2002). During recent years, the circumpolar regions were surveyed as a whole, expanding and updating the previous observations (Doherty et al., 2010; Forsstrom et al., 2009), which includes near one hundred sites and two thousand samples in the Arctic and sub-Arctic regions.

We summarize the spring measurements of present-day (mainly from 2007 to 2009) SBC in the Arctic from all studies known to us. Some of these include vertically-integrated measurements or measurements at various depths throughout the snowpack, and others just include surface measurements. Concentrations supplied by Doherty and others have been increased by $11 \%$ relative to their earlier study (Doherty et al., 2010), in order to correct for a low bias in the concentrationdependent parameters $\left(C_{\mathrm{BC}}^{\max }, C_{\mathrm{BC}}^{\text {est }}\right.$ and $\left.C_{\mathrm{BC}}^{\text {equiv }}\right)$ which resulted from an error in the earlier data analysis. As reported by Doherty et al. (2010), the vertical distribution of sBC in the Russian Arctic is non-uniform, with larger values in the upper $25 \%$ of the snowpack at most measurement sites (left panel in Fig. 1). In contrast, the concentrations observed in the Canadian Arctic are smaller and relatively uniform throughout the snowpack (right panel in Fig. 1). Both sets of vertical profiles involve measurements of $\mathrm{sBC}$ and snow depth from dozens of snow pits, thus they are considered to be largely representative of the typical distribution of spring $\mathrm{SBC}$ in these regions. The typical values of snowpack depth in Russia and Canada were respectively $34 \pm 7 \mathrm{~cm}$ and $30 \pm 14 \mathrm{~cm}$ across all measurement sites. We calculate the surface and subsurface concentrations of $\mathrm{sBC}$ from the observations at 

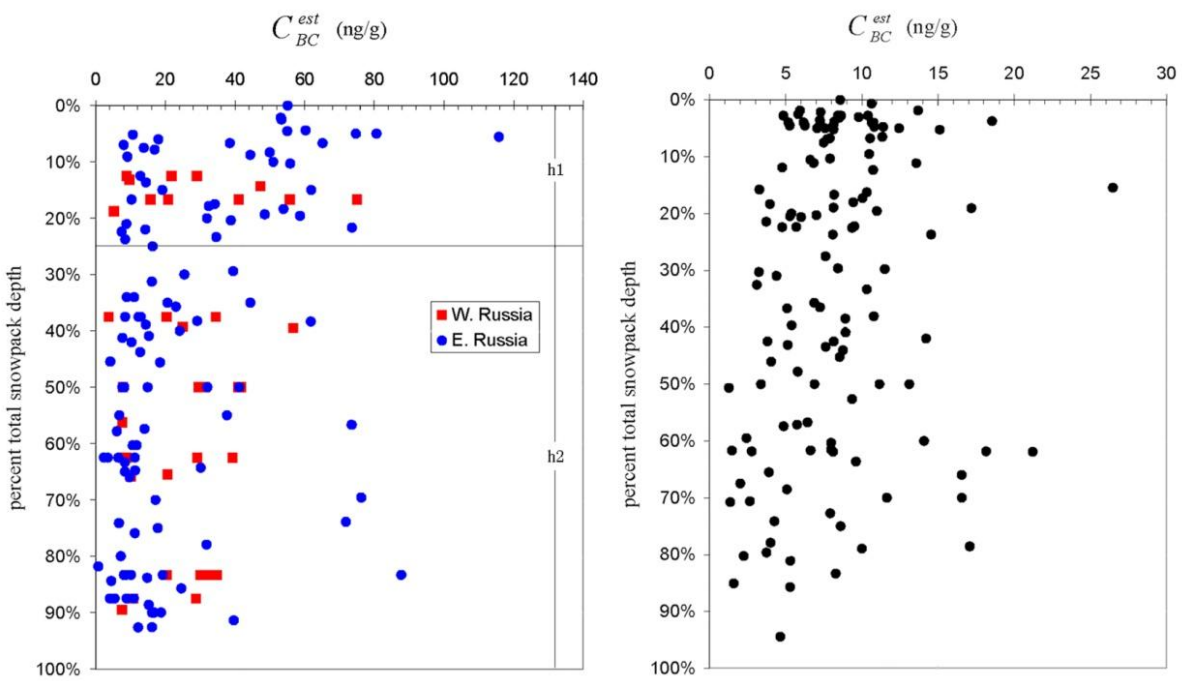

Fig. 1. Vertical profile of sBC concentrations in the Arctic snow from the field measurements in west (2007) and east (2008) Russia (left), and profiles from the field measurements in Canada in spring 2009 (right). $C_{\mathrm{BC}}^{\text {est }}$ denotes the estimated true mass of BC per mass of snow. These two figures are respectively from Fig. 12a and Fig. 9 (left panel) in Doherty et al. (2010), but with some necessary modifications to highlight different concentrations of $\mathrm{sBC}$ in various depths. The snowpack depths in Russia and Canada were respectively $34 \pm 7 \mathrm{~cm}$ and $30 \pm 14 \mathrm{~cm}$ across all measurement sites.

different depths, and then give an equation for estimating the integrated-layer concentration $\left(C_{h}, h=h 1+h 2\right)$ of sBC. In the case of surface $\mathrm{sBC}>$ subsurface $\mathrm{sBC}$ (for most measurement sites in Russian Arctic):

$C_{h}=\frac{25 \% \rho_{h 1} C_{h 1}+75 \% \rho_{h 2} C_{h 2}}{25 \% \rho_{h 1}+75 \% \rho_{h 2}}$

where $C_{h 1}$ is the concentration of sBC in the surface snow $\left(h_{1}=25 \% \cdot h\right)$ at each site, $\rho_{h 1}$ is the average density of surface snow, $C_{h 2}$ is the concentration of sBC in subsurface $\left(h_{2}=75 \% \cdot h\right), \rho_{h 2}$ is the average density of subsurface snow and $h_{1}$ is the top $25 \%$ of the snow pack depth and $h_{2}$ is the bottom $75 \%$. Note that it is difficult to calculate the precise $C_{h}$ based on present limited observations, because we have not obtained the snow density values that correspond to BC measurements in various snow depths. In this study, we apply the estimated snow density of the surface and subsurface layer: $\rho_{h 1}=0.256 \mathrm{~g} \mathrm{~cm}^{-3}$ and $\rho_{h 2}=0.345 \mathrm{~g} \mathrm{~cm}^{-3}$ that is calculated from the mean values of snow density in different types of snow layers observed during the SHEBA campaign (Sturm et al., 2002). Sturm et al. (1995) gave a rough generalization of snow kinds in different terrain types and suggested that the snow found at tundra is the closest substitute for what would be estimated to be found over floating ice. Accordingly, the values of snow density applied in this study are considered to be representative of the typical vertical profiles of snow density in and around the Arctic Ocean in spring. That is generally composed of wind slab, recent and new snow, fine-grained snow, wind slab, and depth hoar from top to bottom by turns (Sturm et al., 2002). In the estimation of total-layer sBC, we presume that the uncertain- ties are entirely represented by the standard deviations of observed snow densities. We take the depth-weighted average of the concentration values in each layer as the estimated vertically-integrated concentration when the value of surface $\mathrm{sBC}$ was close to or less than that of subsurface sBC. All surface and subsurface observations used in this study and the derived vertically-integrated values are listed in Table 1. In order to avoid influence from the surrounding urban environment, Table 1 only includes the estimated values of $\mathrm{sBC}$ in the Arctic Ocean and its adjacent coastal regions, excluding ones far away from the Arctic Ocean. The verticallyintegrated values larger than $70 \mathrm{ng} \mathrm{g}^{-1}$ taken in some sites, were also excluded because large difference between surface and subsurface concentrations was due to local contamination, which may bring great uncertainties. The concentrations of sBC shown in Table 1 have been measured either by the "spectrophotometry" method (for most values in Doherty et al., 2010) or the "thermo-optical NIOSH 5040" method (for the values in this study and Forsstrom et al., 2009). Both of the methods discriminate $\mathrm{BC}$ and non-BC fractions of $\mathrm{ab}$ sorption in the measuring process. It is generally thought that the "spectrophotometry" method is better in the albedo effect analysis. However, in spatial distribution analysis, both of them could imply real variability because discrepancies among measured amounts greatly exceed the estimated errors induced by different measurement methods (Hansen et al., 2004). 
Table 1. Present-day sBC observations in and around the Arctic Ocean in spring. The estimated sBC in this study were calculated from surface and subsurface concentrations with the snow densities obtained in SHEBA field campaign. Most of the surface and subsurface values listed in this table are from Doherty et al. (2010) and others are from several field campaigns known to us. The measurements within a single model grid ("15-20”, “32-33”, “44-45”, “46-49”, "53-63”, “64-70”, "71-73”) are averaged before the comparison with modeled sBC.

\begin{tabular}{|c|c|c|c|c|c|c|c|c|c|c|}
\hline NO. & $\begin{array}{l}\text { Measurement } \\
\text { Region }\end{array}$ & Lat $(\mathrm{N})$ & Lon (E) & $\begin{array}{l}\text { Measured sBC } \\
\text { in surface } \\
\text { snow }\left(\mathrm{ng} \mathrm{g}^{-1}\right)\end{array}$ & $\begin{array}{l}\text { Measured sBC } \\
\text { in subsurface } \\
\text { snow }\left(\mathrm{ng} \mathrm{g}^{-1}\right)\end{array}$ & $\begin{array}{l}\text { Measured sBC } \\
\text { in whole layer } \\
\text { snow }\left(\mathrm{ng} \mathrm{g}^{-1}\right)\end{array}$ & $\begin{array}{l}\text { Measurement } \\
\text { period }\end{array}$ & Reference & $\begin{array}{l}\text { Estimated } \\
\mathrm{sBC}\left(\mathrm{ng} \mathrm{g}^{-1}\right)\end{array}$ & $\begin{array}{l}\text { Uncertainty } \\
\text { of Estimation } \\
\left(\mathrm{ng} \mathrm{g}^{-1}\right)\end{array}$ \\
\hline 1 & Beaufort Sea & 73.000 & 215.000 & 9.94 & 8.63 & - & Apr 2007 & Doherty et al. (2010) & 8.89 & 0.08 \\
\hline 2 & Beaufort Sea & 75.340 & 224.343 & - & - & 8.00 & Apr 2007 & APLIS/SEDNA campaign & 8.00 & - \\
\hline 3 & Arctic Ocean & 82.880 & 205.550 & - & _- & 7.00 & Apr-May 2006 & NPEO (Field campaign) & 7.00 & - \\
\hline 4 & Arctic Ocean & 84.182 & 47.507 & _- & _- & $6.70(\mathrm{n} / \mathrm{a})$ & May 2008 & Doherty et al. (2010) & 6.70 & _- \\
\hline 5 & Arctic Ocean & 84.363 & 62.997 & - & - & $10.90(\mathrm{n} / \mathrm{a})$ & May 2008 & Doherty et al. (2010) & 10.90 & - \\
\hline 6 & Arctic Ocean & 84.700 & 296.520 & _- & _- & 6.00 & Apr-May 2008 & Doherty et al. (2010) & 5.00 & _- \\
\hline 7 & Arctic Ocean & 85.897 & 25.797 & - & - & $5.80(\mathrm{n} / \mathrm{a})$ & May 2008 & Doherty et al. (2010) & 5.80 & - \\
\hline 8 & Arctic Ocean & 86.142 & 45.342 & - & - & $7.10(\mathrm{n} / \mathrm{a})$ & Apr 2008 & Doherty et al. (2010) & 7.10 & - \\
\hline 9 & Arctic Ocean & 86.300 & 334.830 & _- & _- & 6.75 & Apr-May 2008 & Switchyard campaign & 6.75 & _- \\
\hline 10 & Arctic Ocean & 88.133 & 269.283 & - & - & $4.60(\mathrm{n} / \mathrm{a})$ & Spring 2007 & Doherty et al. (2010) & 4.60 & - \\
\hline 11 & Arctic Ocean & 88.345 & 3.407 & _- & _- & $7.20(\mathrm{n} / \mathrm{a})$ & Apr 2008 & Doherty et al. (2010) & 7.20 & - \\
\hline 12 & Arctic Ocean & 88.560 & 314.375 & - & - & 5.00 & Apr-May 2008 & Switchyard campaign & 5.00 & - \\
\hline 13 & Arctic Ocean & 88.850 & 175.433 & - & - & $9.20(\mathrm{n} / \mathrm{a})$ & Apr 2006 & Doherty et al. (2010) & 9.20 & - \\
\hline 14 & N. Pole & 89.150 & 257.517 & _- & _- & $6.00(\mathrm{n} / \mathrm{a})$ & Spring 2007 & Doherty et al. (2010) & 6.00 & _- \\
\hline 15 & N. Pole & 89.300 & 358.120 & - & - & $4.85(\mathrm{n} / \mathrm{a})$ & Spring 2008 & Doherty et al. (2010) & 4.85 & - \\
\hline 16 & N. Pole & 89.400 & 359.000 & _- & _- & $2.33(\mathrm{n} / \mathrm{a})$ & Spring 2008 & Doherty et al. (2010) & 2.33 & - \\
\hline 17 & N. Pole & 89.500 & 358.930 & - & - & $2.51(\mathrm{n} / \mathrm{a})$ & Spring 2008 & Doherty et al. (2010) & 2.51 & - \\
\hline 18 & N. Pole & 89.700 & 336.400 & - & - & 3.00 & Apr-May 2007 & NPEO (Field campaign) & 3.00 & - \\
\hline 19 & N. Pole & 89.900 & 329.400 & _- & _- & $4.20(\mathrm{n} / \mathrm{a})$ & Spring 2008 & Doherty et al. (2010) & 4.20 & _- \\
\hline 20 & N. Pole & 90.000 & 0.980 & - & - & $4.00(\mathrm{n} / \mathrm{a})$ & Spring 2008 & Doherty et al. (2010) & 4.00 & - \\
\hline 21 & Canadian Arctic & 66.171 & 255.626 & 13.90 & 6.68 & - & Spring 2009 & Doherty et al. (2010) & 8.11 & 0.46 \\
\hline 22 & Canadian Arctic & 67.878 & 283.530 & 9.40 & 4.20 & - & Spring 2009 & Doherty et al. (2010) & 5.23 & 0.33 \\
\hline 23 & Canadian Arctic & 68.305 & 255.913 & 6.97 & 10.30 & - & Spring 2009 & Doherty et al. (2010) & 9.64 & 0.21 \\
\hline 24 & Canadian Arctic & 68.568 & 230.477 & 9.10 & 6.57 & _- & Spring 2009 & Doherty et al. (2010) & 7.07 & 0.16 \\
\hline 25 & Canadian Arctic & 68.824 & 264.711 & 12.40 & 7.30 & - & Spring 2009 & Doherty et al. (2010) & 8.31 & 0.32 \\
\hline 26 & Canadian Arctic & 68.986 & 224.938 & 7.15 & 11.55 & _- & Spring 2009 & Doherty et al. (2010) & 10.68 & 0.28 \\
\hline 27 & Canadian Arctic & 69.280 & 282.954 & 8.60 & 5.13 & - & Spring 2009 & Doherty et al. (2010) & 5.82 & 0.22 \\
\hline 28 & Alaskan Arctic & 69.300 & 216.200 & 5.00 & - & - & Spring 2009 & Doherty et al. (2010) & 5.00 & - \\
\hline 29 & Canadian Arctic & 69.635 & 227.819 & 10.00 & 9.00 & - & Spring 2009 & Doherty et al. (2010) & 9.20 & 0.06 \\
\hline 30 & Canadian Arctic & 69.663 & 250.904 & 7.10 & 5.70 & - & Spring 2009 & Doherty et al. (2010) & 5.98 & 0.09 \\
\hline 31 & Canadian Arctic & 69.895 & 247.253 & 6.00 & 13.87 & _- & Spring 2009 & Doherty et al. (2010) & 12.31 & 0.50 \\
\hline 32 & Canadian Arctic & 70.067 & 235.027 & 15.90 & 10.50 & - & Spring 2009 & Doherty et al. (2010) & 11.57 & 0.34 \\
\hline 33 & Canadian Arctic & 70.067 & 235.027 & 12.10 & 5.30 & - & Spring 2009 & Doherty et al. (2010) & 6.65 & 0.43 \\
\hline 34 & Canadian Arctic & 71.151 & 280.752 & 4.70 & 3.43 & _- & Spring 2009 & Doherty et al. (2010) & 3.68 & 0.08 \\
\hline 35 & Alaskan Arctic & 71.325 & 203.567 & 9.00 & - & - & Spring 2009 & Doherty et al. (2010) & 9.00 & - \\
\hline 36 & Canadian Arctic & 72.341 & 277.645 & 9.50 & 3.24 & _- & Spring 2009 & Doherty et al. (2010) & 4.48 & 0.40 \\
\hline 37 & Canadian Arctic & 72.566 & 259.193 & 15.20 & 6.30 & - & Spring 2009 & Doherty et al. (2010) & 8.06 & 0.57 \\
\hline 38 & Canadian Arctic & 75.497 & 263.855 & 9.97 & 9.40 & _- & Spring 2009 & Doherty et al. (2010) & 9.51 & 0.04 \\
\hline 39 & Canadian Arctic & 76.555 & 255.268 & 9.32 & 8.65 & - & Spring 2009 & Doherty et al. (2010) & 8.78 & 0.04 \\
\hline 40 & Canadian Arctic & 76.633 & 263.788 & 11.70 & 3.80 & - & Spring 2009 & Doherty et al. (2010) & 5.37 & 0.50 \\
\hline 41 & Canadian Arctic & 76.867 & 274.786 & 9.20 & 5.83 & _- & Spring 2009 & Doherty et al. (2010) & 6.50 & 0.21 \\
\hline 42 & Canadian Arctic & 80.083 & 273.300 & 12.00 & - & - & Spring 2007 & Doherty et al. (2010) & 12.00 & - \\
\hline 43 & Western Russia & 67.631 & 53.646 & 19.00 & 8.00 & _- & Spring 2007 & Doherty et al. (2010) & 10.18 & 0.70 \\
\hline 44 & Western Russia & 73.381 & 81.429 & 17.60 & 21.65 & - & Spring 2007 & Doherty et al. (2010) & 20.85 & 0.26 \\
\hline 45 & Western Russia & 73.428 & 81.481 & 12.00 & 27.00 & - & Spring 2007 & Doherty et al. (2010) & 24.03 & 0.95 \\
\hline 46 & Western Russia & 72.176 & 102.839 & 83.60 & 34.20 & - & Spring 2007 & Doherty et al. (2010) & 44.00 & 3.14 \\
\hline 47 & Western Russia & 72.211 & 102.933 & 23.20 & 27.90 & - & Spring 2007 & Doherty et al. (2010) & 26.97 & 0.30 \\
\hline 48 & Western Russia & 72.277 & 103.102 & 45.80 & 40.40 & - & Spring 2007 & Doherty et al. (2010) & 41.47 & 0.34 \\
\hline 49 & Western Russia & 72.244 & 103.019 & 62.10 & 40.90 & _- & Spring 2007 & Doherty et al. (2010) & 45.10 & 1.35 \\
\hline 50 & Eastern Russia & 72.054 & 128.523 & 87.90 & 17.60 & - & Spring 2008 & Doherty et al. (2010) & 31.54 & 4.47 \\
\hline 51 & Eastern Russia & 74.065 & 128.872 & 13.00 & 26.00 & - & Spring 2008 & Doherty et al. (2010) & 23.42 & 0.83 \\
\hline 52 & Eastern Russia & 71.649 & 127.894 & 82.00 & 25.80 & - & Spring 2008 & Doherty et al. (2010) & 36.94 & 3.57 \\
\hline 53 & Eastern Russia & 68.631 & 160.369 & 83.20 & 15.50 & - & Spring 2008 & Doherty et al. (2010) & 28.92 & 4.30 \\
\hline 54 & Eastern Russia & 68.649 & 160.487 & 80.00 & 14.00 & _- & Spring 2008 & Doherty et al. (2010) & 27.09 & 4.20 \\
\hline 55 & Eastern Russia & 68.663 & 160.592 & 88.30 & 18.50 & - & Spring 2008 & Doherty et al. (2010) & 32.34 & 4.44 \\
\hline 56 & Eastern Russia & 69.043 & 161.122 & 55.60 & 23.50 & - & Spring 2008 & Doherty et al. (2010) & 29.87 & 2.04 \\
\hline 57 & Eastern Russia & 69.044 & 161.123 & 53.15 & 19.90 & - & Spring 2008 & Doherty et al. (2010) & 26.49 & 2.11 \\
\hline 58 & Eastern Russia & 69.032 & 161.201 & 50.00 & 26.00 & - & Spring 2008 & Doherty et al. (2010) & 30.76 & 1.53 \\
\hline 59 & Eastern Russia & 69.019 & 161.278 & 39.30 & 18.37 & - & Spring 2008 & Doherty et al. (2010) & 22.52 & 1.33 \\
\hline 60 & Eastern Russia & 69.020 & 161.279 & 52.40 & 31.90 & - & Spring 2008 & Doherty et al. (2010) & 35.97 & 1.30 \\
\hline 61 & Eastern Russia & 68.737 & 161.521 & 57.70 & 15.53 & _- & Spring 2008 & Doherty et al. (2010) & 23.89 & 2.68 \\
\hline 62 & Eastern Russia & 68.719 & 161.572 & 53.00 & 18.00 & - & Spring 2008 & Doherty et al. (2010) & 24.94 & 2.23 \\
\hline 63 & Eastern Russia & 68.700 & 161.623 & 42.05 & 8.30 & - & Spring 2008 & Doherty et al. (2010) & 14.99 & 2.15 \\
\hline 64 & Eastern Russia & 69.869 & 169.302 & 11.00 & 10.00 & - & Spring 2008 & Doherty et al. (2010) & 10.20 & 0.06 \\
\hline 65 & Eastern Russia & 69.782 & 169.720 & 11.90 & 8.07 & - & Spring 2008 & Doherty et al. (2010) & 8.83 & 0.24 \\
\hline 66 & Eastern Russia & 68.930 & 170.713 & 14.30 & 9.30 & - & Spring 2008 & Doherty et al. (2010) & 10.29 & 0.32 \\
\hline 67 & Eastern Russia & 69.330 & 170.856 & 11.50 & 15.30 & - & Spring 2008 & Doherty et al. (2010) & 14.55 & 0.24 \\
\hline 68 & Eastern Russia & 69.119 & 170.858 & 14.00 & 11.00 & - & Spring 2008 & Doherty et al. (2010) & 11.59 & 0.19 \\
\hline 69 & Eastern Russia & 69.022 & 170.918 & 23.20 & 10.80 & - & Spring 2008 & Doherty et al. (2010) & 13.26 & 0.79 \\
\hline 70 & Eastern Russia & 69.195 & 170.946 & 13.20 & 12.00 & - & Spring 2008 & Doherty et al. (2010) & 12.24 & 0.08 \\
\hline 71 & Eastern Russia & 69.571 & 171.015 & 9.50 & 14.88 & _- & Spring 2008 & Doherty et al. (2010) & 13.81 & 0.34 \\
\hline 72 & Eastern Russia & 69.524 & 171.310 & 13.00 & 13.00 & - & Spring 2008 & Doherty et al. (2010) & 13.00 & 0.00 \\
\hline 73 & Eastern Russia & 69.478 & 171.605 & 18.00 & 14.43 & - & Spring 2008 & Doherty et al. (2010) & 15.14 & 0.23 \\
\hline 74 & Western Svalbard & 78.910 & 11.720 & - & - & 3.20 & Mar-Apr 2007 & Forsstrom et al. (2009) & 3.20 & - \\
\hline 75 & Western Svalbard & 78.870 & 12.460 & - & - & 1.70 & Apr 2007 & Forsstrom et al. (2009) & 1.70 & - \\
\hline 76 & Western Svalbard & 79.000 & 14.000 & - & - & 1.40 & Apr 2007 & Forsstrom et al. (2009) & 1.40 & - \\
\hline 77 & Eastern Svalbard & 77.897 & 18.302 & - & - & 9.80 & Mar 2007 & Forsstrom et al. (2009) & 9.80 & - \\
\hline 78 & Eastern Svalbard & 78.750 & 17.580 & - & _- & 6.60 & Mar 2007 & Forsstrom et al. (2009) & 6.60 & - \\
\hline 79 & Eastern Svalbard & 79.910 & 25.000 & - & - & 6.50 & Apr 2007 & Forsstrom et al. (2009) & 6.50 & - \\
\hline 80 & Northern Greenland & 81.000 & 301.000 & - & - & $2.60(\mathrm{n} / \mathrm{a})$ & Spring 2008 & Doherty et al. (2010) & 2.60 & - \\
\hline 81 & West of Greenland & 77.450 & 299.500 & - & _- & $3.30(\mathrm{n} / \mathrm{a})$ & Spring 2008 & Doherty et al. (2010) & 3.30 & - \\
\hline 82 & West of Greenland & 76.40 & 292.30 & - & - & $4.30(\mathrm{n} / \mathrm{a})$ & Spring 2008 & Doherty et al. (2010) & 4.30 & - \\
\hline
\end{tabular}


Table 2. Ratios of wet deposition to wet plus dry deposition in the GISS model from September to May 2007-2009.

\begin{tabular}{lrrrrrrrrr}
\hline & Sep & Oct & Nov & Dec & Jan & Feb & Mar & Apr & May \\
\hline North of $66^{\circ} \mathrm{N}$ & 0.95 & 0.94 & 0.92 & 0.91 & 0.90 & 0.90 & 0.92 & 0.93 & 0.92 \\
Northern Hemisphere & 0.78 & 0.78 & 0.77 & 0.76 & 0.74 & 0.75 & 0.76 & 0.77 & 0.76 \\
Worldwide & 0.81 & 0.81 & 0.81 & 0.81 & 0.81 & 0.82 & 0.82 & 0.82 & 0.81 \\
\hline
\end{tabular}

\section{Model description}

The model GISS-PUCCINI is the NASA Goddard Institute for Space Studies (GISS) atmospheric composition and climate model. It consists of the model for Physical Understanding of Composition-Climate Interactions and Impacts (PUCCINI) (Shindell et al., 2006), which is fully embedded in the GISS modelE climate model (Schmidt et al., 2006). The atmospheric model version used here, GISS-E2, is that used for the Coupled Model Intercomparison Project (CMIP) phase 5 simulations in support of the IPCC fifth assessment report (AR5), and contains updates to the physics relevant to aerosols, including the ability to represent multiple downdrafts and updrafts in convective systems, while the black carbon model is unchanged from Koch and Hansen (2005). The model was run at $2^{\circ}$ latitude by $2.5^{\circ}$ longitude horizontal resolution with 40 vertical layers. Simulations were performed in a chemical transport model-like mode for 19952009 using observed sea surface temperatures (Rayner et al., 2003) and linear relaxation of winds towards either NCEP or MERRA reanalysis (Kalnay et al., 1996; Rienecker et al., 2011) to facilitate comparison with observations. Results are analyzed for 2006-2009. Repeating year 2000 monthlyvarying emissions were used from the data set assembled for the AR5 simulations (Lamarque et al., 2010), with the exception of biomass burning emissions which were monthly- and annually-varying emissions from the Global Fire Emission Database (GFED) version 3 (van der Werf et al., 2010). The emissions are respectively $3.5{\mathrm{Tg} \mathrm{r}^{-1}}^{-1}$ from biomass burning and $5.3 \mathrm{Tg} \mathrm{yr}^{-1}$ from fossil fuel and biofuel combustion. In this model, wet deposition depends upon solubility and on transport within convective plumes, scavenging within and below updrafts, rainout within both convective and largescale clouds, washout below precipitating regions, evaporation of falling precipitation, and both detrainment and evaporation from convective plumes. Wet deposition is the dominant removal process in this model. Its fraction of total deposition worldwide and over the Arctic has been shown in Table 2, from which we can see the values worldwide and over the Arctic regions are consistent with those given in other models of $78 \%$ to $>95 \%$ (Textor et al., 2006; Huneeus et al., 2011). Dry deposition is calculated using a resistance-inseries model coupled to a global, seasonally varying vegetation dataset. More details are available in (Koch et al., 1999; Shindell et al., 2001).

\section{Initial field in the Arctic Ocean}

Another issue that needs to be considered is the initial snow cover (depth and density) and sBC distribution over multiyear sea ice at the beginning of the accumulation and deposition season (in this study - September). The initial field represents the snow and $\mathrm{BC}$ content within it that survived melt season. Because thermodynamic and dynamic models of sea ice cover are limited in their ability to simulate the time-varying ice conditions during the summer, we assume the initial snow cover to be that of the September climatological snow conditions in Warren et al. (1999), which was also applied in the estimation of snow depth in Kwok Kwok and Cunningham (2008). The observations of snow and ice density (recent snow: $0.102 \pm 0.019 \mathrm{~g} \mathrm{~cm}^{-3}$ surface $[<3 \mathrm{~cm}]: 0.291 \pm 0.056 \mathrm{~g} \mathrm{~cm}^{-3}$ subsurface $[>3 \mathrm{~m}]$ : $0.333 \pm 0.02 \mathrm{~g} \mathrm{~cm}^{-3}$ ) in the third Chinese Arctic expedition route $\left(79.8-85.4^{\circ} \mathrm{N}, 144.1-170.1^{\circ} \mathrm{W}\right)$ in summer 2008 have been applied in the estimation of snow water equivalent (SWE) and in the calculation of vertically- integrated concentrations of $\mathrm{sBC}$ from surface and subsurface measurements. In this study, we maintain a separate record of the initial conditions at each grid cell. Table 3 summarizes the original observations and estimated values of $\mathrm{sBC}$ in the Arctic Ocean in summer 2005, 2008 and 2010. These measurements range from $74^{\circ} \mathrm{N}$ to $89^{\circ} \mathrm{N}$, all of them are used to represent the climatological distribution of sBC over the multiyear sea ice in summer. The entire Arctic Ocean is divided into three latitudinal bands- south of $80^{\circ} \mathrm{N}, 80-85^{\circ} \mathrm{N}$ and the Arctic Ocean center, the distribution of sBC in each band is represented by the mean values of the observations located within it. In this case, the initial fields are not time-varying and are identical for the results shown in this work.

\section{Intercomparison between model results and observations}

Present observations of sBC show sketchy but identifiable variation in spatial distribution, with maximum values in the Russian Arctic and much lower values in other regions. From the simulations, calculated from the model dry and wet deposition in spring 2007-2009, we can see that model values are also higher over the Eurasian than the Canadian and Alaskan Arctic, followed by the Arctic Ocean, with the lowest in Greenland (Fig. 2). There is an apparent gradient from around the coast to the central Arctic Ocean, 
Table 3. Present-day sBC observations in the Arctic Ocean in summer. The estimated sBC of whole layer at each measurement site is calculated from surface and subsurface BC concentrations with snow densities observed in the 3rd Chinese Arctic expedition in summer 2008. Samples were collected on the sea ice surface, of melting sea ice, aged snow and newly-fallen snow from late July through late September. Thus, there are large uncertainties in current analysis, and present observations can only give a general and rough indication of the spatial distribution of sBC in summer. As the initial field of BC deposition, the Arctic Ocean is divided into several latitude zones in this study and each zone is characterized by one value averaged from the observations within this area.

\begin{tabular}{|c|c|c|c|c|c|c|c|c|c|}
\hline NO. & Lat $(\mathrm{N})$ & Lon $(\mathrm{E})$ & $\begin{array}{l}\text { Measured sBC } \\
\text { in surface } \\
\text { snow }\left(\mathrm{ng} \mathrm{g}^{-1}\right)\end{array}$ & $\begin{array}{l}\text { Measured sBC } \\
\text { in subsurface } \\
\text { snow }\left(\mathrm{ng} \mathrm{g}^{-1}\right)\end{array}$ & $\begin{array}{l}\text { Measured sBC } \\
\text { in whole layer } \\
\text { snow }\left(\mathrm{ng} \mathrm{g}^{-1}\right)\end{array}$ & $\begin{array}{l}\text { Measurement } \\
\text { period }\end{array}$ & Reference & $\begin{array}{l}\text { Estimated } \\
\mathrm{sBC}\left(\mathrm{ng} \mathrm{g}^{-1}\right)\end{array}$ & $\begin{array}{l}\text { Uncertainty of } \\
\text { Estimation } \\
\left(\mathrm{ng} \mathrm{g}^{-1}\right)\end{array}$ \\
\hline 1 & 73.7 & 192.59 & - & - & 5.7 & Jul 2010 & This study & 5.7 & - \\
\hline 2 & 73.72 & 192.72 & - & - & 6.5 & Jul 2010 & This study & 6.5 & - \\
\hline 3 & 73.74 & 193.04 & - & - & 15.9 & Jul 2010 & This study & 15.9 & - \\
\hline 4 & 75 & 200.01 & - & - & 11.4 & Jul 2010 & This study & 11.4 & - \\
\hline 5 & 75 & 202.5 & - & - & 2.8 & Jul 2010 & This study & 2.8 & - \\
\hline 6 & 75.02 & 199.98 & - & - & 2.9 & Jul 2010 & This study & 2.9 & - \\
\hline 7 & 75.03 & 200.52 & - & - & 7.4 & Jul 2010 & This study & 7.4 & - \\
\hline 8 & 75.71 & 222.8 & - & - & 16 & Aug 2005 & Perovich et al. (2009) & 16 & - \\
\hline 9 & 75.908 & 219.411 & 10.2 & 10.2 & - & Aug 2008 & Doherty et al. (2010) & 10.2 & 0.0 \\
\hline 10 & 75.908 & 219.411 & 12.2 & 8.5 & - & Aug 2008 & Doherty et al. (2010) & 9.7 & 0.2 \\
\hline 11 & 75.99 & 203.05 & - & - & 41.6 & Jul 2010 & This study & 41.6 & - \\
\hline 12 & 76 & 203.97 & - & - & 10.2 & Jul 2010 & This study & 10.2 & - \\
\hline 13 & 76.81 & 199.48 & - & - & 40.2 & Aug 2010 & This study & 40.2 & - \\
\hline 14 & 77.04 & 200.18 & - & - & 22.1 & Aug 2010 & This study & 22.1 & - \\
\hline 15 & 77.04 & 200.18 & - & - & 9.5 & Aug 2010 & This study & 9.5 & - \\
\hline 16 & 77.04 & 200.18 & - & - & 8.5 & Aug 2010 & This study & 8.5 & - \\
\hline 17 & 77.702 & 213.398 & 10 & - & - & Jul 2008 & Doherty et al. (2010) & 10.0 & - \\
\hline 18 & 77.9 & 200.13 & - & - & 39.2 & Aug 2010 & This study & 39.2 & - \\
\hline 19 & 77.98 & 200.36 & - & - & 29.8 & Aug 2010 & This study & 29.8 & - \\
\hline 20 & 77.98 & 200.36 & - & - & 10.8 & Aug 2010 & This study & 10.8 & - \\
\hline 21 & 78 & 220.42 & - & - & $15(\mathrm{n} / \mathrm{a})$ & Aug 2008 & Doherty et al. (2010) & 15.0 & - \\
\hline 22 & 78.01 & 209.818 & 4.2 & 15.2 & - & Aug 2008 & Doherty et al. (2010) & 14.3 & 0.6 \\
\hline 23 & 78.291 & 183.321 & 12.3 & - & - & Aug 2005 & Doherty et al. (2010) & 12.3 & - \\
\hline 24 & 78.387 & 206.549 & 21 & 22.1 & - & Aug 2008 & Doherty et al. (2010) & 21.6 & 0.1 \\
\hline 25 & 78.392 & 206.377 & 16.4 & 9.3 & - & Aug 2008 & Doherty et al. (2010) & 10.9 & 0.4 \\
\hline 26 & 78.6 & 216.5 & - & - & 26 & Aug 2005 & Perovich et al. (2009) & 26.0 & - \\
\hline 27 & 79.83 & 331.21 & - & - & $1.2(\mathrm{n} / \mathrm{a})$ & Aug 2008 & Doherty et al. (2010) & 1.2 & - \\
\hline 28 & 79.88 & 333.99 & - & - & $4.8(\mathrm{n} / \mathrm{a})$ & Aug 2008 & Doherty et al. (2010) & 4.8 & - \\
\hline 29 & 79.988 & 209.713 & 28.9 & - & - & Aug 2008 & Doherty et al. (2010) & 28.9 & - \\
\hline 30 & 80.003 & 209.656 & 49.4 & - & - & Aug 2008 & Doherty et al. (2010) & 49.4 & - \\
\hline 31 & 80.081 & 209.792 & 23.5 & 13.7 & - & Aug 2008 & Doherty et al. (2010) & 18.3 & 0.6 \\
\hline 32 & 81.226 & 182.805 & 3.5 & - & - & Aug 2005 & Doherty et al. (2010) & 3.5 & - \\
\hline 33 & 81.226 & 182.805 & 4.1 & - & - & Aug 2005 & Doherty et al. (2010) & 4.1 & - \\
\hline 34 & 81.723 & 209.035 & 5.7 & 8.6 & - & Aug 2008 & Doherty et al. (2010) & 7.2 & 0.2 \\
\hline 35 & 81.926 & 210.071 & 14.1 & - & - & Aug 2008 & Doherty et al. (2010) & 14.1 & - \\
\hline 36 & 81.997 & 219.943 & 4.3 & 17.7 & - & Aug 2008 & Doherty et al. (2010) & 14.4 & 0.8 \\
\hline 37 & 83.087 & 185.329 & 3 & - & - & Aug 2005 & Doherty et al. (2010) & 3.0 & - \\
\hline 38 & 83.087 & 185.329 & 4.3 & - & - & Aug 2005 & Doherty et al. (2010) & 4.3 & - \\
\hline 39 & 83.299 & 188.112 & 1.8 & - & - & Aug 2005 & Doherty et al. (2010) & 1.8 & - \\
\hline 40 & 83.299 & 188.112 & 5.2 & - & - & Aug 2005 & Doherty et al. (2010) & 5.2 & - \\
\hline 41 & 83.955 & 216.808 & 4.2 & - & - & Aug 2005 & Doherty et al. (2010) & 4.2 & - \\
\hline 42 & 83.955 & 216.808 & 11.8 & - & - & Aug 2005 & Doherty et al. (2010) & 11.8 & - \\
\hline 43 & 84.171 & 209.005 & 2.6 & - & - & Aug 2005 & Doherty et al. (2010) & 2.6 & - \\
\hline 44 & 84.171 & 209.005 & 7 & - & - & Aug 2005 & Doherty et al. (2010) & 7.0 & - \\
\hline 45 & 84.307 & 210.918 & 4.6 & - & - & Aug 2005 & Doherty et al. (2010) & 4.6 & - \\
\hline 46 & 84.309 & 199.352 & 2 & - & - & Aug 2005 & Doherty et al. (2010) & 2.0 & - \\
\hline 47 & 84.309 & 199.352 & 14.4 & - & - & Aug 2005 & Doherty et al. (2010) & 14.4 & - \\
\hline 48 & 84.311 & 199.581 & 2.2 & - & - & Aug 2005 & Doherty et al. (2010) & 2.2 & - \\
\hline 49 & 84.311 & 199.581 & 15.7 & - & - & Aug 2005 & Doherty et al. (2010) & 15.7 & - \\
\hline 50 & 85.122 & 205.2 & 18.8 & - & - & Sep 2005 & Doherty et al. (2010) & 18.8 & - \\
\hline 51 & 85.938 & 48.335 & 6.4 & 2.8 & - & Sep 2005 & Doherty et al. (2010) & 4.5 & 0.2 \\
\hline 52 & 85.938 & 48.335 & 9.6 & - & - & Sep 2005 & Doherty et al. (2010) & 9.6 & - \\
\hline 53 & 86.657 & 55.618 & 3.7 & 6.1 & - & Sep 2005 & Doherty et al. (2010) & 5.0 & 0.1 \\
\hline 54 & 87.472 & 57.588 & 4.2 & 9.6 & - & Sep 2005 & Doherty et al. (2010) & 7.1 & 0.3 \\
\hline 55 & 87.62 & 155.876 & 2.7 & - & - & Sep 2005 & Doherty et al. (2010) & 2.7 & - \\
\hline 56 & 87.62 & 155.876 & 30 & - & - & Sep 2005 & Doherty et al. (2010) & 30.0 & - \\
\hline 57 & 87.66 & 150.902 & 8 & - & - & Aug 2005 & Perovich et al. (2009) & 8.0 & - \\
\hline 58 & 88.056 & 58.748 & 2.3 & 2.1 & - & Sep 2005 & Doherty et al. (2010) & 2.2 & 0.0 \\
\hline 59 & 88.456 & 146.532 & 5.3 & - & - & Sep 2005 & Doherty et al. (2010) & 5.3 & - \\
\hline 60 & 88.456 & 146.532 & 22.4 & - & - & Sep 2005 & Doherty et al. (2010) & 22.4 & - \\
\hline 61 & 88.46 & 213.47 & 7 & - & - & Aug 2005 & Perovich et al. (2009) & 7.0 & - \\
\hline 62 & 88.813 & 164.136 & 18.2 & - & - & Sep 2005 & Doherty et al. (2010) & 18.2 & - \\
\hline 63 & 88.813 & 164.136 & 1.7 & - & - & Sep 2005 & Doherty et al. (2010) & 1.7 & - \\
\hline 64 & 89.374 & 270.912 & 2.7 & 13.3 & - & Sep 2005 & Doherty et al. (2010) & 8.4 & 0.6 \\
\hline 65 & 89.482 & 169.798 & 7.1 & 2.3 & - & Sep 2005 & Doherty et al. (2010) & 4.5 & 0.3 \\
\hline
\end{tabular}




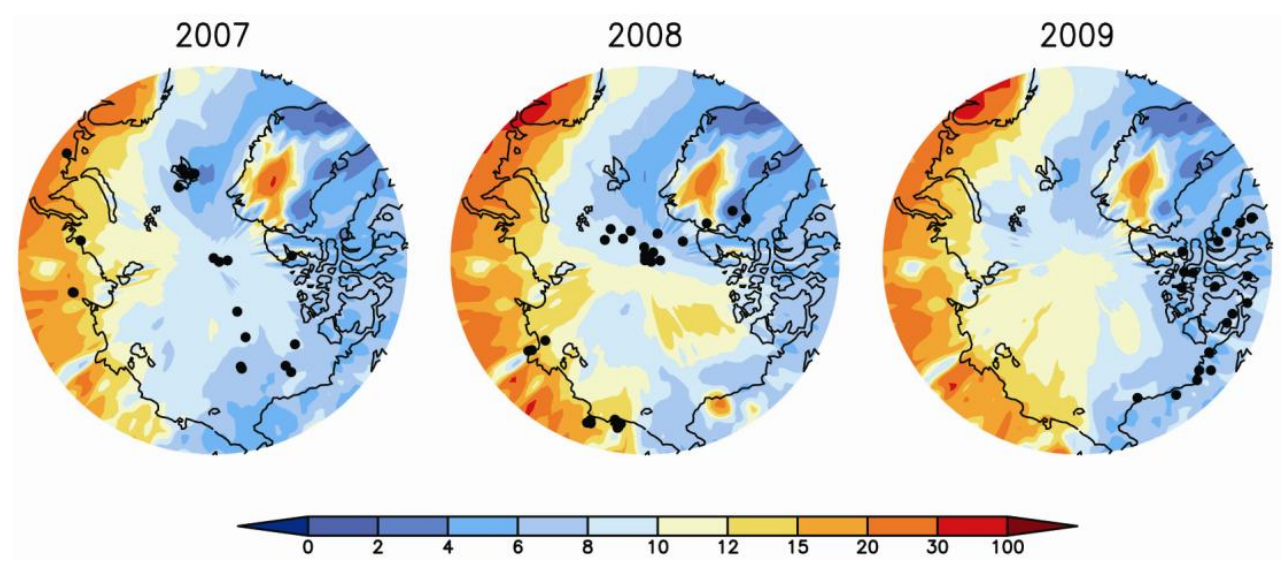

Fig. 2. Simulated sBC in and around the Arctic Ocean in spring 2007-2009. Black circles indicate the measurement sites for the corresponding years.

especially in the Russian Arctic, but relatively little variability over the Arctic Ocean. The mean concentration of sBC over the Arctic Ocean shows large interannual variability in spring from 2007 to 2009 (7.8-13.4 $\left.\mathrm{ng} \mathrm{g}^{-1}\right)$. Point to point comparisons between measured sBC and model simulations from NCEP and MERRA runs have been carried out respectively in the Arctic Ocean and surrounding seas, the Canadian and Alaskan Arctic, the Russian Arctic, Svalbard and Greenland (Fig. 3). Model results have been interpolated to each measurement site in the corresponding month and year of observations and are compared with the mean observed value in cases where more than one measurement is available within a $2 \times 2.5$ degree grid box. From Fig. 4 , it can be seen that both of the simulated values from two model runs are comparable with most of present observations over each Arctic sub-region. Rough agreement between the model and observations is found in the Arctic Ocean and Canadian and Alaskan Arctic sector, respectively with mean values of $8.2 \pm 0.9 \mathrm{ng} \mathrm{g}^{-1}$ and $7.2 \pm 1.3 \mathrm{ng} \mathrm{g}^{-1}$ in the model and $7.4 \pm 2.3 \mathrm{ng} \mathrm{g}^{-1}$ and $7.8 \pm 2.4 \mathrm{ng} \mathrm{g}^{-1}$ in the observations. The concentrations of modeled sBC in Western Greenland in spring 2008 are also close to the observations; mean values are $3.8 \pm 1.8 \mathrm{ng} \mathrm{g}^{-1}$ in the model and $3.8 \pm 0.7 \mathrm{ng} \mathrm{g}^{-1}$ in the observations. However, there is an apparent difference in Northern Greenland, which conforms to the abnormal peakvalue in the central inland areas of Greenland (Fig. 3). More measurements would be needed to determine the actual distribution of sBC there. Additionally, the values of modeled sBC are significantly smaller than observations in the Russian Arctic sector in spring 2007. Previous studies indicate that anthropogenic influence was dominant in western Russia in spring 2007, and open fire influence was dominant in Eastern Russia in spring 2008 (Doherty et al., 2010; Wang et al., 2011). Indeed, the modeled values in Eastern Russia are substantially higher in 2008 than in 2007, perhaps owing to the interannually varying biomass burning emissions. Hence an underestimation of biomass burning emissions in Russia may be the main reason of this simulation bias.

The simulation values in Svalbard are within the range of observations and near to the median value. Analysis of previous measurements (81 samples) in Svalbard indicates that there exist obvious spatial variations, the concentrations of $\mathrm{sBC}$ in eastern Svalbard are significantly higher than the western side (Forsstrom et al., 2009) and the emissions from the western European Arctic may be dominant in this distribution pattern. This spatial variation has also been reproduced by the model even though the modeled concentrations in western Svalbard are a little higher than the observed concentrations.

Further study on the simulation from two model runs shows small difference over each Arctic sector. NCEP run performs slightly better in the Arctic Ocean, Svalbard and Greenland, MERRA run performs slightly better in Russia, Canadian and Alaskan Arctic, although they both show a significant underestimation in the Russian Arctic, and overestimation in Svalbard and Greenland (Table 4). The difference between the two model runs was far less than current simulation bias. Therefore, we suggest that the discrepancy between model results and observations comes from other sources and not from different meteorology fields applied.

We also estimated the decrease in snow and sea ice albedo and resulting radiative forcing based on the SBC distribution simulated by the current GISS-PUCCINI model. Figure 4 shows the albedo reduction in and around the Arctic Ocean in spring 2007-2009 due to BC deposition. We estimated the impact of sBC on snow and ice albedo according to the "spectrally averaged" albedo change by BC content illustrated in Fig. 2 in Warren and Wiscombe (1985) and assume the snow grain radius is a constant of $100 \mu \mathrm{m}$ (McConnell et al., 2007) with no significant aging. The resulting albedo reduction presents significant space-time variations, with highest mean value of $1.25 \%$ in the Russian 


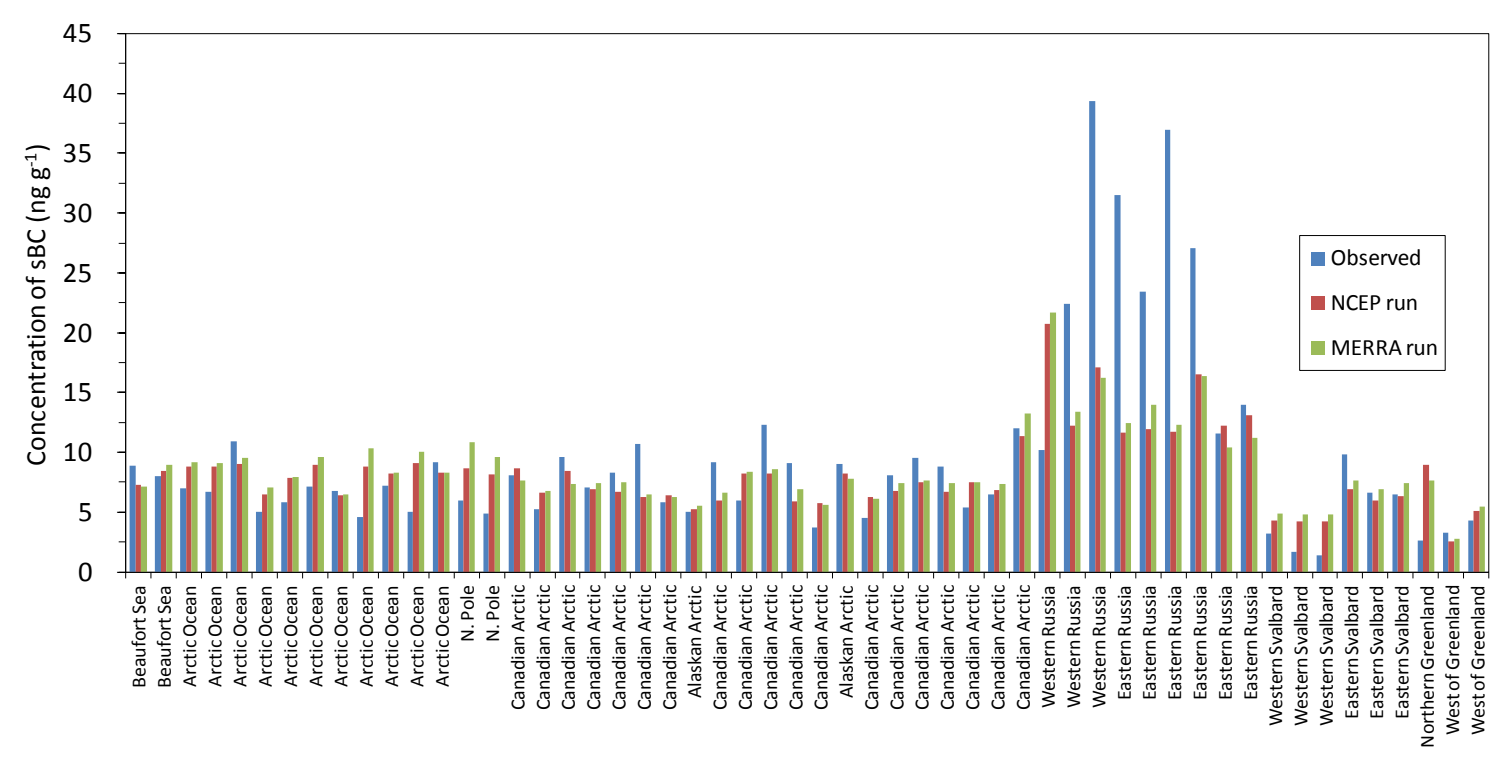

Fig. 3. Histogram of modeled versus observed sBC for the Arctic Ocean and surrounding regions in spring 2007-2009. Model results are sampled for the month and year of observations and interpolated to corresponding measurement sites by bilinear interpolation methodology. Simulated values from NCEP and MERRA runs are shown both.

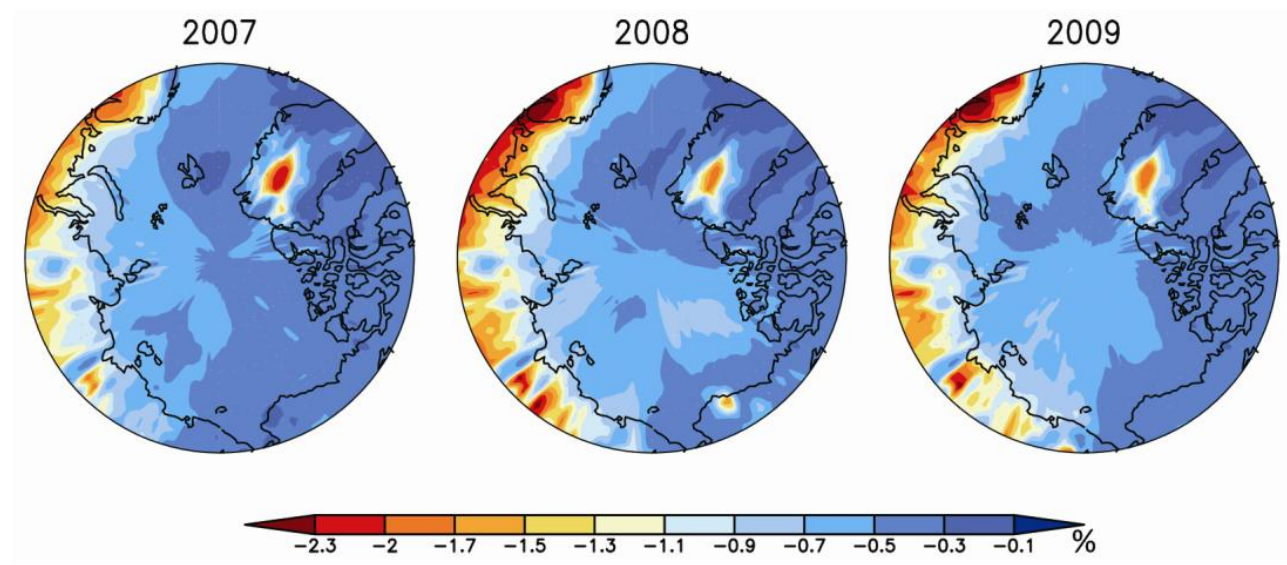

Fig. 4. Modeled change in snow and ice albedo due to sBC in and around the Arctic Ocean (north to $66^{\circ} \mathrm{N}$ ) in spring $2007-2009$.

Arctic, which was much larger than those in other Arctic regions ranging $0.39 \%$ to $0.64 \%$. The averaged value over the Arctic north of $66^{\circ} \mathrm{N}$ is $0.4-0.6 \%$ from spring 2007-2009, lower than the estimates of Park et al. (2005), but comparable with Jacobson et al. (2004). On the basis of this result of albedo decrease, we evaluate the radiative forcing caused by $\mathrm{sBC}$ with the NCEP downwelling surface solar radiation. Result shows that the radiative forcings from $\mathrm{BC}$ deposition to the Arctic snow and ice (north to $66^{\circ} \mathrm{N}$ ) are $0.7 \mathrm{~W} \mathrm{~m}^{-2}$, $1.1 \mathrm{~W} \mathrm{~m}^{-2}$ and $1.0 \mathrm{~W} \mathrm{~m}^{-2}$, respectively in spring 2007,2008 and 2009. Wang et al. (2011) reported a radiative forcing of $1.2 \mathrm{~W} \mathrm{~m}^{-2}$ for spring 2007-2009 in the Arctic north to $60^{\circ} \mathrm{N}$, similar to Flanner et al. (2007) and slightly larger than our values.
Finally, we review the research on the potential source type/region of Arctic BC in previous studies. Rahn and McCaffrey (1980) reported that the Arctic atmosphere is hazy in winter and spring, and indicated this phenomenon may be caused by fossil fuel burning, industrial and agricultural processes, by long-range transport of mid-latitude pollution products. Generally, Europe, North America and South Asia are considered to be the main contributors to present Arctic sBC, while contributions from biomass burning sources are also significant (Hegg et al., 2009). Matsui et al. (2011) suggests that biomass burning and anthropogenic sources in high-latitude were most important for the Arctic BC both in spring and summer, because most of $\mathrm{BC}$ from lowerlatitude regions has been removed by wet deposition before arriving at the Arctic. Among these source regions, Europe 
Table 4. Observation-to-model ratios between observations and model results from NCEP and MERRA runs over each Arctic sector.

\begin{tabular}{lccccc}
\hline & Arctic Ocean & Canada and Alaska & Russia & Svalbard & Greenland \\
\hline NCEP & $\mathbf{0 . 9 2}$ & 1.09 & 1.81 & $\mathbf{0 . 8 4}$ & $\mathbf{0 . 8 1}$ \\
MERRA & 0.89 & $\mathbf{1 . 0 5}$ & $\mathbf{1 . 7 7}$ & 0.73 & 0.78 \\
\hline
\end{tabular}

contributes more than $50 \%$ of the Arctic BC loading mainly through transport within the lower troposphere in all seasons (Huang et al., 2010b), which is the most likely cause of the highest concentrations in the Russian Arctic and surrounding Seas. Eleftheriadis et al. (2009) found some differences in the seasonal contribution to the BC measured at Svalbard by Eurasian sources with the BC peak values mainly during the winter arriving from Siberian and Western Russian sources, while during the warm season North Europe including West Russia was the main contributing area. Hegg et al. (2009) also indicates that local emissions from fossil-fuel combustion make a significant contribution to the springtime highlatitude Arctic Ocean and some locations in Western Russia. North America contributes about $10-20 \%$ to the Arctic troposphere with least variations in the contribution from different vertical levels (Huang et al., 2010b; Shindell et al., 2008), and is also regarded as a main biomass source to Greenland, the Alaskan and the Canadian Arctic (McConnell et al., 2007; Hegg et al., 2009). Because of its remoteness from the main source regions, the concentrations in Greenland and the central Arctic Ocean are much lower than other Arctic regions. South Asia is also considered to be a significant contributor in the Arctic upper troposphere/lower stratosphere during the springtime but it does not appear to contribute significantly to the deposited BC (Koch and Hansen, 2005; Hirdman et al., 2010). In addition to the pollution from long range transport sources, the emissions from ships and cross-polar aircraft flight are also very important. Corbett et al. (2007) indicates that the impacts from local shipping are comparable with long range transport from lower latitude emission sources. Emission form international shipping can be up to 71000-160000 metric tons annually, representing about $2 \%$ of global BC from all sources. It is also shown that $1.2 \mathrm{Gg} \mathrm{BC}$ has been emitted from shipping within the Arctic in 2004, most of which was derived from transit vessels, container and general cargo ships, and the rest from fishing vessels (Corbett et al., 2010a). The vessel activities are mainly concentrated in summer and autumn months, and are increasing dramatically with the rapid decrease of the Arctic sea ice. In this background, many approaches have been recommended to reduce PM emissions from diesel engines and ships, such as switching to low-sulfur fuels and engine process modifications (Corbett et al., 2010b). Cross-polar aircraft flight is also an important contributor to the Arctic BC. Whitt et al. (2011) point out that $2.35 \mathrm{Tg}$ fuel were burned in the Arctic Circle $\left(66.56^{\circ} \mathrm{N}-90^{\circ} \mathrm{N}\right)$ in 2006 and more than half of commercial aviation emissions occur in relatively sta- ble regions of the atmosphere and nearly one quarter occur in the stratosphere. In view of the validation against present spatially extensive observations of $\mathrm{sBC}$, we suggest that the latest NASA GISS-E2-PUCCINI model would be a rational choice to further identify source regions of Arctic BC.

\section{Discussion and conclusions}

There are large uncertainties in current model simulations of BC in the Arctic. Various models give very different regional source contributions and different evaluation of radiative forcing due to $\mathrm{BC}$ deposition. It is useful to characterize model biases by comparing results with present field observations, in order to reduce these uncertainties. In this study, spatially extensive comparisons between model results and present $\mathrm{sBC}$ observations are presented, to validate the latest GISS model deposition of $\mathrm{BC}$ in the Arctic regions. It is demonstrated that the latest GISS model performs well in large scale simulations of $\mathrm{BC}$ deposition, especially in the Arctic Ocean and Canadian Arctic sector. However, there is still apparent underestimation for both runs in a few measurement sites in Russian Arctic. Further comparison of modeled aBC with surface long-term observations at Barrow, Alert, Zeppelin and Nord stations shows that the latest GISS model has been greatly improved in simulating the seasonal variations of the Arctic BC (Fig. 5), in comparison to previous version mentioned in Shindell et al. (2008), but still has significant underestimation in winter and spring, especially at Barrow station, where the bias can be up to one order of magnitude. This is in agreement with the results from earlier studies (Koch et al., 2009b; Schwarz et al., 2010), in which comparisons of vertical profiles of simulated BC with corresponding observations had been done over the Arctic Ocean, North American, Canada and Greenland in spring and during a flight line from Anchorage to the north pole (60$80^{\circ} \mathrm{N}$ ) in winter. The results indicated that the simulated vertical profiles were comparable with observations in Greenland, Canada and Arctic Ocean in spring. However, there was significant bias in Alaska and nearby regions whether in spring or winter, with the model underestimating BC concentrations in the middle and lower troposphere. It is generally thought that several factors may be the cause of this bias. Earlier studies pointed out that the lack of seasonal variations from current simulations may be due to poorly simulated deposition processes rather than transport processes (Schwarz et al., 2010; Huang et al., 2011; Quinn et al., 2011). Liu et al. (2011) found that winter concentrations of BC 

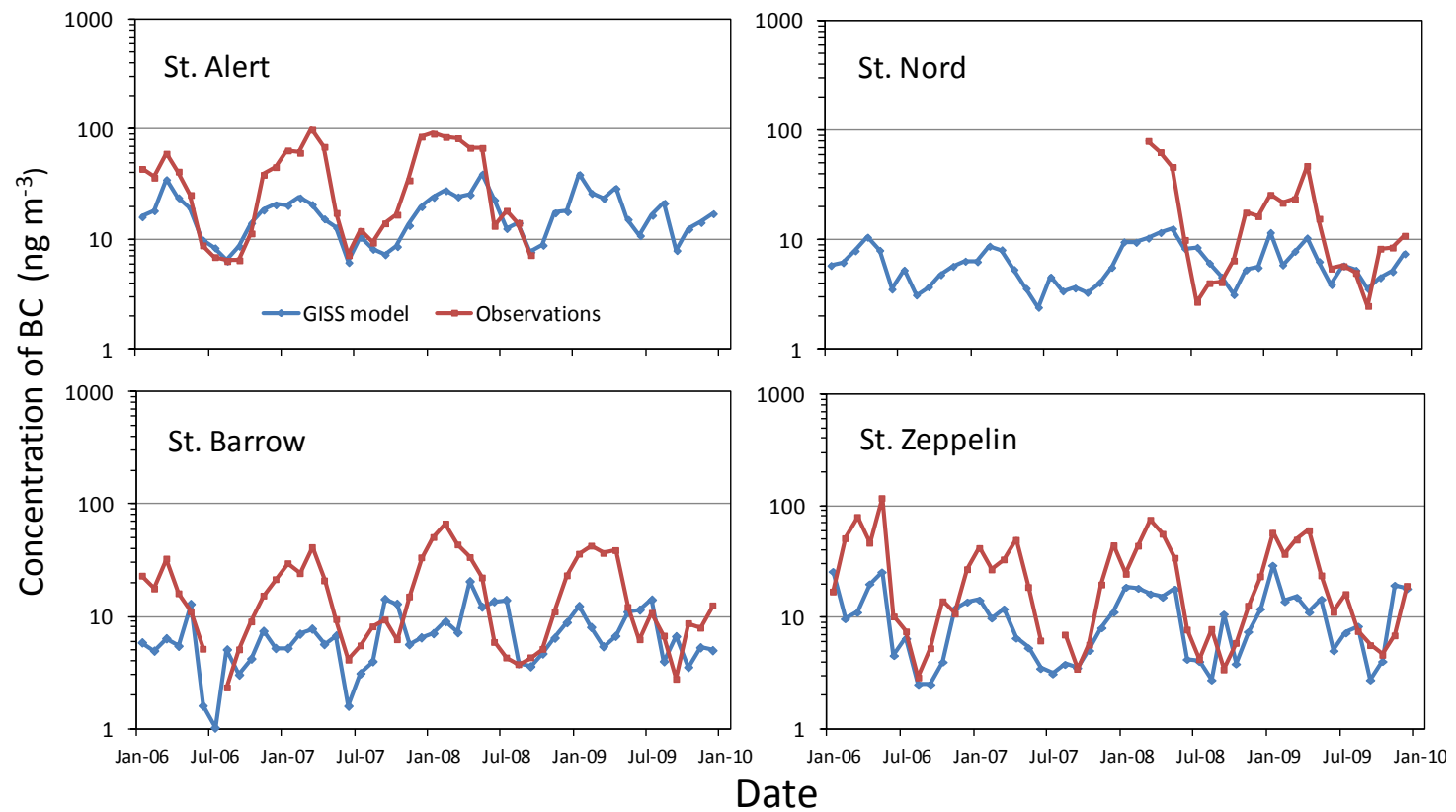

Fig. 5. Comparison of modeled monthly aBC concentrations in near surface layer versus measured concentrations for $\mathrm{Alert}\left(83^{\circ} \mathrm{N}, 62^{\circ} \mathrm{W}\right)$, Barrow $\left(71^{\circ} \mathrm{N}, 157^{\circ} \mathrm{W}\right)$, Zeppelin $\left(79^{\circ} \mathrm{N}, 12^{\circ} \mathrm{E}\right)$ and Nord $\left(81^{\circ} \mathrm{N}, 16^{\circ} \mathrm{W}\right)$ station from January 2006 to December 2009.

in the Arctic could increase throughout much of the tropospheric column by improving parameterization of $\mathrm{BC}$ aging and deposition processesin the model. Huang et al. (2011) suggested that the dry deposition process has little effect on the seasonal pattern of $\mathrm{BC}$ in the Arctic lower troposphere, the observed seasonality of $\mathrm{BC}$ in the Arctic troposphere is mainly due to the seasonal changes in aerosol removal by wet scavenging and seasonal injections of $\mathrm{BC}$ from Europe and the former USSR. Koch et al. (2009) also suggested that current simulation bias in Arctic BC may be due to lack of sufficient removal by precipitating clouds and low-level poleward transport. Therefore, we expect current model output could perhaps be enhanced by improving the physical parameterization of wet deposition processes and biomass burning emissions in the Arctic. Of course, this still needs to be verified by a series of sensitivity tests with the model. However, present $\mathrm{BC}$ observations, obtained by means of various measurement technologies, also have large uncertainties (Koch et al., 2009). In addition, aircraft measurements were actually carried out during short flight campaigns, which basically provide a "snapshot" of vertical profiles of BC concentrations. This makes the comparison with simulated monthly $\mathrm{BC}$ distribution quite challenging since one emission event from a forest or grass fire in the region, could modify the vertical BC profile and greatly affect the results of aircraft measurements.

It is especially worth noting that the largest simulation biases of sBC appeared in the Russian Arctic (see Fig. 3). Although there are a few measurement sites included in the comparison from this region, we expect that biomass burn- ing emissions may be the main cause of the poor performance here. On the other hand, Arctic industrial emissions could also play a role and could have changed over the 2000s decade. There are two pieces of evidence to support this assumption: (1) biomass burning and anthropogenic sources in high-latitude were regarded as the most important contributor to the Arctic BC (Matsui et al., 2011), and emission from biomass burning is dominant in the contribution to Arctic sBC (Hegg et al., 2009). (2) Strong cooling of the air over the snow cover in Eurasia allows polluted air from northern Eurasia to penetrate the entire Arctic at low altitudes (Stohl et al., 2006), and Northern Eurasia is most likely to be the source region of the $\mathrm{aBC}$ in the lower troposphere in the Arctic in winter and early spring (Quinn et al., 2011). Final conclusions cannot be reached from the above analyses. Further model simulations of $\mathrm{BC}$ are needed to determine if emissions are responsible, and if so to show which type of emissions from what regions and which type of sources lead to the current bias found in the Russian Arctic. For example, running the model in a period when Russian forest fires were minimal could provide some insight. In addition to the inherent problems in the model, the approximations and hypotheses used in the calculation of vertically-integrated concentration of sBC may be another reason for the underestimation. We applied climatological observations of snow density obtained in the SHEBA campaign to simulate the actual values of snow density in different depths at each measurement site and this may lead to much larger uncertainties in the results obtained for the Russian Arctic compared with other Arctic regions, as significant variations in the vertical profiles 
are observed there. More measurements of snow density in various snow depths are needed to reduce this uncertainty. Finally, more measurements with large spatial coverage in the Arctic would also be particularly useful for model improvement. For instance, more aircraft observations for BC are needed over Eurasia, the oceans and the biomass burning regions.

Acknowledgements. We especially thank S. J. Doherty who supplied most of the sBC observations and gave valuable comments on this study. We would like to thank the anonymous referee for their valuable comments greatly improving the paper. We also would like to thank Chinese Arctic and Antarctic Administration for providing the opportunities of participating in the Arctic expeditions and the kindly help of all staff in the 3rd Chinese Arctic expedition and the 1st Korean Arctic expedition. We also acknowledge Sharma Sangeeta and Anne Jefferson for sharing long-term BC observations at Alert and Barrow station, and Joshua Schwarz for his positive help in the comparison of vertical profiles of BC from GISS model with aircraft measurement data obtained during HIPPO1 campaign. Discussion with Q. Wang has been very valuable for the estimation of albedo reduction. The concentrations of $\mathrm{sBC}$ reported in this study are measured in Center for Atmosphere Watch and Service, Chinese Academy of Meteorological Sciences. This work was funded by the Opening Founding of State Key Laboratory of Cryospheric Sciences (SKLCS 09-07), National Basic Research Program of China (2011CB403401, 2010CB955608), NSFC(41121001), and key project of CAMS (2010Z002).

Edited by: T. Wagner

\section{References}

Baumgardner, D., Popovicheva, O., Allan, J., Bernardoni, V., Cao, J., Cavalli, F., Cozic, J., Diapouli, E., Eleftheriadis, K., Genberg, P. J., Gonzalez, C., Gysel, M., John, A., Kirchstetter, T. W., Kuhlbusch, T. A. J., Laborde, M., Lack, D., Müller, T., Niessner, R., Petzold, A., Piazzalunga, A., Putaud, J. P., Schwarz, J., Sheridan, P., Subramanian, R., Swietlicki, E., Valli, G., Vecchi, R., and Viana, M.: Soot reference materials for instrument calibration and intercomparisons: a workshop summary with recommendations, Atmos. Meas. Tech., 5, 1869-1887, doi:10.5194/amt-51869-2012, 2012.

Clarke, A. and Noone, K.: Soot in the Arctic: A cause for perturbation in radiative transfer, J. Geophys. Res., 19, 2045-2053, 1985.

Corbett, J. J., Wang, C., Winebrake, J. J., and Green, E.: Review of Marpol Annex VI and the NOx Technical Code: Allocation and Forecasting of Global Ship Emissions, International Maritime Organization, London, UK, 27 pp., 2007.

Corbett, J. J., Lack, D. A., Winebrake, J. J., Harder, S., Silberman, J. A., and Gold, M.: Arctic shipping emissions inventories and future scenarios, Atmos. Chem. Phys., 10, 9689-9704, doi:10.5194/acp-10-9689-2010, 2010a.

Corbett, J. J., Winebrake, J., and Green, E.: An assessment of technologies for reducing regional short-lived climate forcers emitted by ships with implications for Arctic shipping, Carbon Management, 1, 207-225, 2010b.
Doherty, S. J., Warren, S. G., Grenfell, T. C., Clarke, A. D., and Brandt, R. E.: Light-absorbing impurities in Arctic snow, Atmos. Chem. Phys., 10, 11647-11680, doi:10.5194/acp-1011647-2010, 2010.

Eleftheriadis, K., Vratolis, S., and Nyeki, S.: Aerosol black carbon in the European Arctic: Measurements at Zeppelin station, Ny-Ålesund, Svalbard from 1998-2007, Geophys. Res. Lett., 36, L02809, doi:10.1029/2008GL035741, 2009.

Flanner, M. G., Zender, C. S., Randerson, J. T., and Rasch, P. J.: Present-day climate forcing and response from black carbon in snow, J. Geophys. Res., 112, D11202, doi:10.1029/2006JD008003, 2007.

Forsstrom, S., Strom, J., Pedersen, C. A., Isaksson, E., and Gerland, S.: Elemental carbon distribution in Svalbard snow, J. Geophys. Res., 114, D19112, doi:10.1029/2008JD011480, 2009.

Grenfell, T., Light, B., and Sturm, M.: Spatial distribution and radiative effects of soot in the snow and sea ice during the SHEBA experiment, J. Geophys. Res., 107, 8032, doi:10.1029/2000JC000414, 2002.

Hansen, J. and Nazarenko, L.: Soot climate forcing via snow and ice albedos, P. Natl. Acad. Sci. USA, 101, 423-428, doi:10.1073/PNAS.2237157100, 2004.

Hansen, J., Sato, M., Ruedy, R., Kharecha, P., Lacis A., Miller, R. L., Nazarenko, L., Lo, K., Schmidt, G. A., Russell, G., Aleinov, I., Bauer, S., Baum, E., Cairns, B., Canuto, V., Chandler, M., Cheng, Y., Cohen, A., Del Genio, A., Faluvegi, G., Fleming, E., Friend, A., Hall, T., Jackman, C., Jonas, J., Kelley, M., Kiang, N. Y., Koch, D., Labow, G., Lerner, J., Menon, S., Novakov, T., Oinas, V., Perlwitz, J. P., Perlwitz, J., Rind, D., Romanou, A., Schmunk, R., Shindell, D., Stone, P., Sun, S., Streets, D., Tausnev, N., Thresher, D., Unger, N., Yao, M., and Zhang, S.: Climate simulations for 1880-2003 with GISS modelE, Clim. Dynam., 29, 661-696., doi:10.1007/s00382-007-0255-8, 2007.

Hegg, D. A., Warren, S. G., Grenfell, T. C., Doherty, S. J., Larson, T. V., and Clarke, A. D.: Source attribution of black carbon in Arctic snow, Environ. Sci. Technol, 43, 4016-4021, 2009.

Hirdman, D., Sodemann, H., Eckhardt, S., Burkhart, J. F., Jefferson, A., Mefford, T., Quinn, P. K., Sharma, S., Ström, J., and Stohl, A.: Source identification of short-lived air pollutants in the Arctic using statistical analysis of measurement data and particle dispersion model output, Atmos. Chem. Phys., 10, 669-693, doi:10.5194/acp-10-669-2010, 2010.

Huang, L., Gong, S. L., Jia, C. Q., and Lavoue, D.: Importance of deposition process in simulating the seasonality of the Arctic black carbon aerosol, J. Geophys. Res., 115, D17207, doi:10.1029/2009JD013478, 2010a.

Huang, L., Gong, S. L., Jia, C. Q., and Lavoue, D.: Relative contributions of anthropogenic emissions to black carbon aerosol in the Arctic, J. Geophys. Res., 115, D19208, doi:10.1029/2009JD013592, 2010b.

Huneeus, N., Schulz, M., Balkanski, Y., Griesfeller, J., Prospero, J., Kinne, S., Bauer, S., Boucher, O., Chin, M., Dentener, F., Diehl, T., Easter, R., Fillmore, D., Ghan, S., Ginoux, P., Grini, A., Horowitz, L., Koch, D., Krol, M. C., Landing, W., Liu, X., Mahowald, N., Miller, R., Morcrette, J.-J., Myhre, G., Penner, J., Perlwitz, J., Stier, P., Takemura, T., and Zender, C. S.: Global dust model intercomparison in AeroCom phase I, Atmos. Chem. Phys., 11, 7781-7816, doi:10.5194/acp-11-7781-2011, 2011. 
Intergovernmental Panel on Climate Change: IPCC Fourth Assessment Report, Climate Change 2007: The Scientific Basis, Cambridge Univ. Press, New York, 2007.

Jacobson M. Z.: Strong radiative heating due to the mixing state of black carbon in atmospheric aerosols, Nature, 409, 695-697, doi:10.1038/35055518, 2001.

Jacobson, M. Z.: The climate response of fossil-fuel and biofuel soot, accounting for soot's feedback to snow and sea ice albedo and emissivity, J. Geophys. Res., 109, D21201, doi:10.1029/2004JD004945, 2004.

Kalnay, E., Kanamitsu, M., Kistler, R., Collins, W., Deaven, D., Gandin, L., Iredell, M., Saha, S., White, G., Woollen, J., Zhu, Y., Leetmaa, A., Reynolds, R., Chelliah, M., Ebisuzaki, W., Higgins, W., Janowiak, J., Mo, K. C., Ropelewski, C., Wang, J., Jenne, R., and Joseph, D.: The NCEP/NCAR 40-year Reanalysis Project, B. Am. Meteorol. Soc., 77, 437-471, 1996.

Koch, D. and Hansen, J.: Distant origins of Arctic black carbon: A Goddard Institute for Space Studies ModelE experiment, J. Geophys. Res., 110, D04204, doi:10.1029/2004JD005296, 2005.

Koch, D., Jacob, D., Tegen, I., Rind, D., and Chin, M.: Tropospheric sulfur simulation and sulfate direct radiative forcing in the Goddard Institute for Space Studies general circulation model, J. Geophys. Res., 104, 23799-23822, 1999.

Koch, D., Menon, S., Del Genio, A., Ruedy, R., Aleinov, I., and Schmidt, G. A.: Distinguishing aerosol impacts on climate over the past century, J. Climate, 22, 2659-2677, 2009a.

Koch, D., Schulz, M., Kinne, S., McNaughton, C., Spackman, J. R., Balkanski, Y., Bauer, S., Berntsen, T., Bond, T. C., Boucher, O., Chin, M., Clarke, A., De Luca, N., Dentener, F., Diehl, T., Dubovik, O., Easter, R., Fahey, D. W., Feichter, J., Fillmore, D., Freitag, S., Ghan, S., Ginoux, P., Gong, S., Horowitz, L., Iversen, T., Kirkevåg, A., Klimont, Z., Kondo, Y., Krol, M., Liu, X., Miller, R., Montanaro, V., Moteki, N., Myhre, G., Penner, J. E., Perlwitz, J., Pitari, G., Reddy, S., Sahu, L., Sakamoto, H., Schuster, G., Schwarz, J. P., Seland, Ø., Stier, P., Takegawa, N., Takemura, T., Textor, C., van Aardenne, J. A., and Zhao, Y.: Evaluation of black carbon estimations in global aerosol models, Atmos. Chem. Phys., 9, 9001-9026, doi:10.5194/acp-9-9001-2009, $2009 b$.

Kwok, R. and Cunningham, G. F.: ICESat over Arctic sea ice: Estimation of snow depth and ice thickness, J. Geophys. Res., 113, C08010, doi:10.1029/2008JC004753, 2008.

Lamarque, J.-F., Bond, T. C., Eyring, V., Granier, C., Heil, A., Klimont, Z., Lee, D., Liousse, C., Mieville, A., Owen, B., Schultz, M. G., Shindell, D., Smith, S. J., Stehfest, E., Van Aardenne, J., Cooper, O. R., Kainuma, M., Mahowald, N., McConnell, J. R., Naik, V., Riahi, K., and van Vuuren, D. P.: Historical (1850-2000) gridded anthropogenic and biomass burning emissions of reactive gases and aerosols: methodology and application, Atmos. Chem. Phys., 10, 7017-7039, doi:10.5194/acp10-7017-2010, 2010.

Liu, J, Fan, S., Horowitz, L. W., and Levy II, H., February: Evaluation of factors controlling long-range transport of black carbon to the Arctic, J. Geophys. Res., 116, D04307, doi:10.1029/2010JD015145, 2011.

Matsui, H., Kondo, Y., Moteki, N., Takegawa, N., Sahu, L. K., Zhao, Y., Fuelberg, H. E., Sessions, W. R., Diskin, G., Blake, D. R., Wisthaler, A., and Koike, M.: Seasonal variation of the transport of black carbon aerosol from the Asian continent to the Arctic during the ARCTAS aircraft campaign, J. Geophys. Res., 116, D05202, doi:10.1029/2010JD015067, 2011.

McConnell, J. R., Edwards, R., Kok, G. L., Flanner, M. G., Zender, C. S., Saltzman, E. S., Banta, J. R., Pasteris, D. R., Carter, M. M., and Kahl, J. D. W.: 20th-Century Industrial Black Carbon Emissions Altered Arctic Climate Forcing, Science, 317, 13811384 doi:10.1126/Science.1144856, 2007.

Park, R. J., Jacob, D. J., Palmer, P. I., Clarke, A. D., Weber, R. J., Zondlo, M. A., Eisele, F. L., Bandy, A. R., Thornton, D. C., Sachse, G. W., and Bond, T. C.: Export efficiency of black carbon aerosol in continental outflow: global implications, J. Geophys. Res.-Atmos., 110, D11205, doi:10.1029/2004JD005432, 2005.

Perovich, D. K., Grenfell, T. C., Light, B., Elder, B. C., Harbeck, J., Polashenski, C., Tucker III, W. B., and Stelmach, C.: Transpolar observations of the morphological properties of Arctic sea ice, J. Geophys. Res., 114, C00A04, doi:10.1029/2008JC004892, 2009.

Quinn, P. K., Bates, T. S., Baum, E., Doubleday, N., Fiore, A. M., Flanner, M., Fridlind, A., Garrett, T. J., Koch, D., Menon, S., Shindell, D., Stohl, A., and Warren, S. G.: Short-lived pollutants in the Arctic: their climate impact and possible mitigation strategies, Atmos. Chem. Phys., 8, 1723-1735, doi:10.5194/acp8-1723-2008, 2008.

Quinn, P. K., Stohl, A., Arneth, A., Berntsen, T., Burkhart, J. F., Christensen, J., Flanner, M., Kupiainen, K., Lihavainen, H., Shepherd, M., Shevchenko, V., Skov, H., and Vestreng, V.: The impact of black carbon on Arctic climate, Arctic Monitoring and Assessment Programme (AMAP), Oslo, 72 pp., 2011.

Rahn, K. A., and McCaffrey, R. J.: On the origin and transport of the winter arctic aerosol, Annals of the New York Academy of Sciences, 338, 486-503, 1980.

Rayner, N. A., Parker, D. E., Horton, E. B., Folland, C. K., Alexander, L. V., Rowell, D. P., Kent, E. C., and Kaplan, A.: Global analyses of sea surface temperature, sea ice, and night marine air temperature since the late nineteenth century, J. Geophys. Res., 108, 4407, doi:10.1029/2002JD002670, 2003.

Rienecker, M. M., Suarez, M. J., Gelaro, R., Todling, R., Bacmeister, J., Liu, E., Bosilovich, M. G., Schubert, S. D., Takacs, L., Kim, G., Bloom, S., Chen, J., Collins, D., Conaty, A., Silva, A. D., Gu, W., Joiner, J., Koster, R. D., Lucchesi, R., Molod, A., Owens, T., Pawson, S., Pegion, P., Redder, C. R., Reichle, R., Robertson, F. R., Ruddick, A. G., Sienkiewicz, M., and Woollen, J.: MERRA: NASA's Modern-Era Retrospective Anal. Res. Appl., J. Climate, 24, 3624-3648, 2011.

Schmidt, G. A., Ruedy, R., Hansen, J. E., Aleinov, I., Bell, N., Bauer, M., Bauer, S., Cairns, B., Canuto, V., Cheng, Y., Genio, A. D., Faluvegi, G., Friend, A. D., Hall, T. M., Hu, Y., Kelley, M., Kiang, N. Y., Koch, D., Lacis, A. A., Lerner, J., Lo, K. K., Miller, R. L., Nazarenko, L., Oinas, V., Perlwitz, J., Perlwitz, J., Rind, D., Romanou, A., Russell, G. L., Sato, M., Shindell, D. T., Stone, P. H., Sun, S., Tausney, N., Thresher, D., and Yao, M.: Present-day atmospheric simulations using GISS ModelE: Comparison to in situ, satellite, and reanalysis data, J. Climate, 19, 153-192, 2006.

Schwarz, J. P., Spackman, J. R., Gao, R. S., Watts, L. A., Stier, P., Schulz, M., Davis, S. M., Wofsy, S. C., and Fahey, D. W.: Global scale black carbon profiles observed in the remote atmosphere and compared to models, Geophys. Res. Lett., 37, L18812, doi:10.1029/2010GL044372, 2010. 
Shindell, D. T., Grenfell, J. L., Rind, D., Price, C., and Grewe, V.: Chemistry climate interactions in the Goddard Institute for Space Studies general circulation model 1. Tropospheric chemistry model description and evaluation, J. Geophys. Res., 106, 8047-8076, 2001.

Shindell, D. T., Faluvegi, G., Unger, N., Aguilar, E., Schmidt, G. A., Koch, D. M., Bauer, S. E., and Miller, R. L.: Simulations of preindustrial, present-day, and 2100 conditions in the NASA GISS composition and climate model G-PUCCINI, Atmos. Chem. Phys., 6, 4427-4459, doi:10.5194/acp-6-4427-2006, 2006.

Shindell, D. T., Chin, M., Dentener, F., Doherty, R. M., Faluvegi, G., Fiore, A. M., Hess, P., Koch, D. M., MacKenzie, I. A., Sanderson, M. G., Schultz, M. G., Schulz, M., Stevenson, D. S., Teich, H., Textor, C., Wild, O., Bergmann, D. J., Bey, I., Bian, H., Cuvelier, C., Duncan, B. N., Folberth, G., Horowitz, L. W., Jonson, J., Kaminski, J. W., Marmer, E., Park, R., Pringle, K. J., Schroeder, S., Szopa, S., Takemura, T., Zeng, G., Keating, T. J., and Zuber, A.: A multi-model assessment of pollution transport to the Arctic, Atmos. Chem. Phys., 8, 5353-5372, doi:10.5194/acp-85353-2008, 2008.

Stohl, A.: Characteristics of atmospheric transport into the arctic troposphere. Journal of Geophysical Research, 111, D11306, doi:10.1029/2005JD006888, 2006.

Sturm, M., Holmgren, J., and Liston, G. E.: A seasonal snow cover classification system for local to global applications, J. Geophys. Res., 8, 1261-1283, 1995.

Sturm, M., Holmgren, J., and Perovich, D. K.: Winter snow cover on the sea ice of the Arctic Ocean at the Surface Heat Budget of the Arctic Ocean (SHEBA): Temporal evolution and spatial variability, J. Geophys. Res., 107, 8047, doi:10.1029/2000JC000400, 2002.
Textor, C., Schulz, M., Guibert, S., Kinne, S., Balkanski, Y., Bauer, S., Berntsen, T., Berglen, T., Boucher, O., Chin, M., Dentener, F., Diehl, T., Easter, R., Feichter, H., Fillmore, D., Ghan, S., Ginoux, P., Gong, S., Grini, A., Hendricks, J., Horowitz, L., Huang, P., Isaksen, I., Iversen, I., Kloster, S., Koch, D., Kirkevåg, A., Kristjansson, J. E., Krol, M., Lauer, A., Lamarque, J. F., Liu, X., Montanaro, V., Myhre, G., Penner, J., Pitari, G., Reddy, S., Seland, Ø., Stier, P., Takemura, T., and Tie, X.: Analysis and quantification of the diversities of aerosol life cycles within AeroCom, Atmos. Chem. Phys., 6, 1777-1813, doi:10.5194/acp-6-1777-2006, 2006.

van der Werf, G. R., Randerson, J. T., Giglio, L., Collatz, G. J., Mu, M., Kasibhatla, P. S., Morton, D. C., DeFries, R. S., Jin, Y., and van Leeuwen, T. T.: Global fire emissions and the contribution of deforestation, savanna, forest, agricultural, and peat fires (19972009), Atmos. Chem. Phys., 10, 11707-11735, doi:10.5194/acp10-11707-2010, 2010.

Wang, Q., Jacob, D. J., Fisher, J. A., Mao, J., Leibensperger, E. M., Carouge, C. C., Le Sager, P., Kondo, Y., Jimenez, J. L., Cubison, M. J., and Doherty, S. J.: Sources of carbonaceous aerosols and deposited black carbon in the Arctic in winter-spring: implications for radiative forcing, Atmos. Chem. Phys., 11, 1245312473, doi:10.5194/acp-11-12453-2011, 2011.

Warren, S. G. and Wiscombe, W. J.: Dirty snow after nuclear war, Nature, 313, 467-470, 1985.

Warren, S. G., Rigor, I. G., and Untersteiner, N.: Snow Depth on Arctic Sea Ice, J. Climate, 12, 1814-1829, 1999.

Whitt, D. B., Wilkerson, J. T., Jacobson, M. Z., Naiman, A. D., and Lele, S. K.: Vertical mixing of commercial aviation emissions from cruise altitude to the surface, J. Geophys. Res., 116, D14109, doi:10.1029/2010JD015532, 2011. 